\title{
The acceptability and utility of different diagnostic tests and sample types for the surveillance of trachoma in the Bijagos Islands, Guinea Bissau
}

\author{
Authors: Ramandeep Sahota and Emma Harding-Esch
}

\begin{abstract}
Introduction
Trachoma is the leading infectious cause of blindness worldwide and until recently was hyperendemic in the Bijagos Islands, a remote archipelago of islands off the coast of Guinea Bissau. Once elimination of trachoma has been achieved in the Bijagos Islands, it is imperative that a successful surveillance programme is put in place. The aim of this study was to determine the acceptability and utility of different diagnostic tests and sample types that could be used for trachoma surveillance.
\end{abstract}

\section{Methods}

Semi-structured interviews of community members and key stakeholders, followed by focus group discussions, explored views on experiences with trachoma, examining the eye for clinical signs, taking a conjunctival sample with a cotton bud, taking a blood sample, laboratory testing, health preferences within the community, and the challenges that may be faced by surveillance programmes.

\section{Results and discussion}

Community members expressed dissatisfaction with their healthcare experiences in relation to trachoma and, in some cases, were keen for different procedures that would be more acceptable and useful. In general, community members and stakeholders indicated a preference for the collection of samples that can be tested in the laboratory to detect trachoma infection. Despite this, stakeholders articulated their contentment with best current practice, with a trend among community members to ultimately be happy with whichever intervention would give them good health.

\section{Conclusion}

In this setting, diagnostic tests and sample types used for trachoma surveillance are accepted by communities to a degree. Appropriate sensitisation of communities prior to the implementation of a trachoma programme is crucial. 